\title{
Bridge Condition Modelling and Prediction Using Dynamic Bayesian Belief Networks
}

\author{
M. Imran Rafiq* \\ School of Environment and Technology, University of Brighton, Brighton, UK. \\ Marios K. Chryssanthopoulos
}

Department of Civil and Environmental Engineering, University of Surrey, Guildford, UK.

Saenthan Sathananthan

Rail Solutions, Atkins, Birmingham, UK.

* Corresponding author:

M. Imran Rafiq

School of Engineering and Technology, University of Brighton, Brighton, UK.

Tel: 00441273642385 , Fax: 00441273642285 , e-mail: m.rafiq@brighton.ac.uk

Co-author e-mails: mkchry@surrey.ac.uk, sathananthan.saenthan@atkinsglobal.com 


\begin{abstract}
The development of a condition-based deterioration modelling methodology at bridge group level using Bayesian Belief Network (BBN) is presented in this paper. $\mathrm{BBN}$ is an efficient tool to handle complex inter-dependencies within elements of engineering systems, by means of conditional probabilities specified on a fixed model structure. The advantages and limitations of the BBN for such applications are reviewed by analysing a sample group of masonry bridges on the UK railway infrastructure network. The proposed methodology is then extended to develop a time dependent deterioration model using a Dynamic Bayesian Network. The condition of elements within the selected sample of bridges and a set of conditional probabilities for static and time dependent variables, based on inspection experience, are used as input to the models to yield, in probabilistic terms, overall condition-based deterioration profiles for bridge groups. Sensitivity towards various input parameters, as well as underlying assumptions, on the point-in-time performance and the deterioration profile of the group are investigated. Together with results from 'what if' scenarios, the potential of the developed methodology is demonstrated in relation to the specification of structural health monitoring requirements and the prioritization of maintenance intervention activities.
\end{abstract}

Keywords: Deterioration modelling, Bayesian Belief Network, Maintenance planning, Masonry Arch Bridges

\title{
1. Introduction
}

Engineering systems comprise a series of interconnected elements, which behave in a complex but integrated manner to perform their intended function. As an example, a bridge is a vital element within a transport system, and is itself comprised of relatively smaller elements e.g. beams, deck slab, abutments, etc. Due to their exposure to harsh environmental conditions, and owing to their critical position within the transport infrastructure, effective management of bridges is vital for the availability and reliability of the overall transport system. Deterioration of 
bridge elements and sub-elements may lead to a reduced level of service and, potentially, a lower safety level. Traditionally, element level management approaches have been extrapolated in managing system performance, especially in dealing with changes due to wear and deterioration.

Predicting deterioration is a vital component of modern bridge management systems. It is generally established for individual bridge elements, often with the aid of inspection records, thus leading to an approximate prediction of bridge performance over time. Currently available methods for this purpose include Markov Chain models, Fault Tree models, Fuzzy System models and more recently Bayesian Belief Networks (BBN). Fault and event tree approaches have found applications in the bridge sector over a decade ago, particularly for propagating element failure probabilities to estimate system failure probabilities (treating the bridge as a system) e.g. Sianpur and Adams (1997) and LeBeau and Wadia-Fascetti (2000). These approaches are based on classical system connectivity leading to series, parallel or mixed systems. However, they become computationally cumbersome when applied to large systems and demand input that is not often available (e.g. Khakzad, Khan, and Amyotte, 2011). Moreover, event tree and fault tree models are typically developed for catastrophic failure events, hence may not be appropriate for those bridges that may fail over an extended period of time (Attoh-Okine and Bowers, 2006), primarily due to deterioration and aging effects. On the other hand, causal probabilistic networks have been proposed since the early 1990's for use in infrastructure engineering, as providing a powerful framework for systems analysis (Casciati and Faravelli, 1992). In particular, BBN's offer a compact representation of a joint probability distribution, together with a rigorous formalism for the construction of models relying on probabilistic knowledge. They can be used to calculate system reliability with due consideration to the associated uncertainties (Langseth and Portinale, 2007; Marquez, Neil and Fenton, 2010). 
They have also been used to formally take account of qualitative data and expert opinions for maintenance management purposes (e.g. Celeux, Corset, Lannoy, and Richard, 2006). Moreover, they are capable of handling complex inter-element relationships by means of conditional probabilities. They can address the variation of performance with time and are efficient in updating of performance with new information, all important considerations in a bridge management context, e.g. Rafiq, Chryssanthopoulos, and Onoufriou, (2004) and Straub (2009). BBNs have also been successfully used in risk-based decision support tools for the marine industry (e.g. Friis-Hansen, 2000; Faber, Kroon, Kragh, Bayly and Decosemaeker, 2002).

An extension of the BBN, called Dynamic Bayesian Network (DBN), may be used to analyse problems with time varying domains, e.g. Murphy (2002) and Weber and Jouffe (2003). Straub (2009) developed a DBN based generic framework for stochastic modelling of deterioration processes and demonstrated its advantages in terms of computational efficiency and ease of model updating through its application to fatigue crack growth problems in steel structures. Nielsen and Sorensen (2011) have demonstrated the use of this approach in risk-based inspection planning of offshore wind turbine foundations. This approach is effective when the location of deterioration is spatially fixed at certain locations on the structure, e.g. cracks at critical joints. However, these so called 'hot spots' are not apparent in other structural types, e.g. stone / masonry arch bridges. In such structural systems, the deterioration, which is typically assessed through condition indicators, is more diffuse and spatially spread, with the structural elements working in an integrated manner (with complex interdependencies) to deliver a holistic system performance. This paper presents the development of a condition-based deterioration model for such structural systems using a BBN/DBN methodology. Inspection results from a sample group of UK masonry arch railway bridges serve as input for the BBN model, which is 
used to obtain the distribution for the overall condition of the bridge group. Using DBN the model is then extended to incorporate time dependent characteristics in order to obtain deterioration profiles for the group under different environmental exposure conditions. These profiles together with a risk ranking strategy for the bridge group, as presented in Sathananthan, Onoufriou, and Rafiq, (2008), can be utilised to develop group/stock level risk-based inspection plans (Sathananthan, 2010). The sensitivity of the results towards various assumptions and input parameters is also discussed in this paper.

\section{Bayesian Belief Networks}

\subsection{Definition of $B B N$}

$\mathrm{BBN}$ is a special case of causal networks, that encodes a multivariate statistical distribution function of a set of $n$ random variables $\left(A_{i} ; i=1 \ldots, n\right)$ into a compact yet concise formalism (Pearl, 1988 and Jensen and Nielsen, 2007). The main features of the formalism are a graphical encoding of a set of conditional independence statements and a compact way of representing a joint probability distribution between the random variables. In essence, a BBN consists of a qualitative and a quantitative part.

The qualitative part of the BBN is a directed acyclic graph (DAG) consisting of a set of nodes (corresponding to the random variables, $A_{i}$, respectively) connected through a set of directed links. The variables preceding a link are termed 'parent' variables. The variables at the other end of the link are called 'child' variables. In cases where more than one parent variables $\left(A_{i}\right)$ are associated with a child variable $\left(A_{j}\right)$, these are denoted as $p a\left(A_{j}\right)$.

The conditional independence statements between the nodes are captured through the directed links using the rules of d-separation (Pearl, 1988). Three connection types are defined 
for this purpose, shown in Fig. 1. The serial connection (Fig. 1a) encodes the statement that variable $\mathrm{C}$ is independent of $\mathrm{A}$ given $\mathrm{B}$; however, these are not independent marginally. For example, let A represent the amount of vegetation on an abutment wall, B represent the extent of cracking of the wall and $\mathrm{C}$ represent the condition of the bridge support (which is dependent on abutment wall deterioration). The encoded conditional independence statement implies that in the absence of information regarding the extent of cracking of the abutment wall, the amount of vegetation on the wall can inform the overall support condition, i.e. A and $\mathrm{C}$ are not independent. However, as soon as the extent of cracking of the wall is known, the amount of vegetation becomes irrelevant to the support condition, i.e. C becomes independent of A given B.

Fig. $1 \mathrm{~b}$ represents a diverging connection, encoding the conditions that $\mathrm{B}$ and $\mathrm{C}$ are independent given $\mathrm{A}$, but are not independent marginally. For example, if A represents the environment surrounding a bridge, $\mathrm{B}$ and $\mathrm{C}$ represent the condition of bridge deck and its supporting beams respectively. The diverging connection encodes that a known condition of the bridge deck can support an inference about the surrounding environmental exposure, leading to an inference regarding the condition of beams, hence $\mathrm{B}$ and $\mathrm{C}$ are not independent marginally. However, if the environmental condition becomes available, the bridge deck and beams may be considered independent of each other, i.e. B and C become independent given A.

Fig. 1c represents a converging connection, encoding the condition that $\mathrm{A}$ and $\mathrm{B}$ are marginally independent, however they are not independent given C. Similar to the previous examples, let variables $\mathrm{A}$ and $\mathrm{B}$ represent two abutment walls of a bridge, and $\mathrm{C}$ represent the overall support condition. The converging connection dictates that the condition of wall $\mathrm{A}$ and $\mathrm{B}$ are marginally independent. However, if knowledge about the overall support condition (C) 
becomes available, the condition of wall A can be inferred from knowledge about wall B and vice versa, hence $\mathrm{A}$ and $\mathrm{B}$ are not independent given $\mathrm{C}$.

The quantitative part of the BBN consists of the conditional probabilities between the parent and child variables. In most applications, the random variables are discretely defined using a set of mutually exclusive condition states together with their associated probabilities. Although continuous random variables have been used in some recent studies, this paper is limited to the use of discrete random variables representing the nodes of the BBN. For each child variable, a conditional probability table (CPT) needs to be defined linking condition states of the child to the parent variables.

The set of conditional independence statements allow calculation of the joint probability distribution using recursive factorisation (Pearl, 1988) as follows

$$
P(U)=P\left(A_{1}, \ldots, A_{n}\right)=\prod_{i=1}^{n} P\left(X_{i} \mid p a\left(X_{i}\right)\right)
$$

Eq. 1

where $U$ is the set of variables $\left(A_{1}, \ldots, A_{n}\right)$ within a BBN. The prior probability distribution of any variable (i.e. probability of a variable in the absence of evidence), A, can be calculated by marginalizing other variables out of the joint probability function in Eq. 1. The method of variable elimination can be used for this purpose as described in Jensen and Nielsen (2007). Starting from a set of ' $n$ ' probability tables (describing all the probabilities associated with the variables of a BBN), each variable except $A$ is marginalised out in turn by multiplying all tables from the set with marginalising variable in their domain and placing the resulting table in the set. The results are unaffected by the sequence of variable marginalisation. This can be expressed in a generic form as follows 


$$
P(A)=\sum_{U \backslash\{A\}} P(U)
$$

Eq. 2

where $\sum_{U \backslash\{A\}} P(U)$ represents recursive marginalisation of each $\mathrm{BBN}$ variable (in this case variable $A$ which is excluded from the right-hand-side operation). In many cases, the process of marginalizing variables can be made computationally efficient without having to deal with the full joint probability function (Jensen and Nielsen, 2007).

Bayesian networks are computationally efficient in updating the model when new information regarding the condition state of any variable becomes available, e.g. through inspection, testing or structural health monitoring. Given that a set of new evidence, $e_{j}$, where $j=$ $1, \ldots ., m$, regarding variables in a $\mathrm{BBN}$ becomes available, the joint probability distribution of Eq. 1 becomes $P(U, e)$. The updated BBN model of Eq. 1 will become (Jensen and Nielsen, 2007)

$$
P(U, e)=\prod_{i=1}^{n} P\left(A_{i} \mid p a\left(A_{i}\right)\right) \cdot \prod_{j=1}^{m} e_{j}
$$

Eq. 3

and the updated probability for any variable $A$, given the evidence, $e$, will become

$$
P(A \mid e)=\frac{\sum_{u \backslash\{A\}} P(U, e)}{P(e)}
$$




\subsection{Dynamic Bayesian Networks (DBN)}

A DBN is a special type of BBN, which deals with domains containing recurring networks that evolve over time. In such cases, discrete units of time are introduced and a model is defined for each unit of time. This local model for each unit of time is called a 'time slice'. In turn, these time slices are connected through temporal links to form the complete model. If the time slices are identical and the temporal links stay the same, then such models are termed as DBN. More details on the DBN and various algorithms for use with DBN are presented in detail in Murphy (2002). For this study, the probabilities associated with the temporal links (also termed transition probabilities), as well as the conditional probabilities of the variables in all time slices of the DBN model, are considered constant, leading to a homogeneous DBN model.

An example DBN is shown in Fig 2. The basic network, which consists of variables A, B and $C$ at time $t_{i}$ (i.e. $A\left(t_{i}\right), B\left(t_{i}\right)$ and $\left.C\left(t_{i}\right)\right)$, repeats over time. Since the DBN is assumed homogeneous, $\mathrm{P}\left[\mathrm{A}\left(\mathrm{t}_{\mathrm{i}+1}\right) \mid \mathrm{A}\left(\mathrm{t}_{\mathrm{i}}\right)\right]$ should be equal to $\mathrm{P}\left[\mathrm{A}\left(\mathrm{t}_{\mathrm{i}}\right) \mid \mathrm{A}\left(\mathrm{t}_{\mathrm{i}-1}\right)\right]$, etc. Thus, each time slice model is treated as a separate $\mathrm{BBN}$, and the probabilities of the child variables within the time slice are calculated using the chain rule outlined above.

\section{A BBN model for Masonry Arch Bridges}

For the purpose of maintenance management, most bridge management systems around the world sub-divide a bridge into components (major elements) and sub-components (minor elements). A bridge is generally divided into three major elements, namely, deck, superstructure, and substructure. These are further subdivided into minor elements (also called basic elements), such as deck slab, girders, abutment walls, piers, etc. Regular inspections are carried out to establish their condition / performance over time; typically, the information from such 
inspections is stored within the management system at the basic element level. This information is later used to assess the condition / performance at a bridge level (for maintenance planning), or at a group and/or stock level (for estimating maintenance budgets). Since the relation between the basic elements, the major elements, and that of the bridge or group of bridges is complex, a $\mathrm{BBN}$ framework has potentially much to offer, since inter-dependencies can be modelled through conditional probabilities.

In this study, a BBN model is developed for masonry arch bridges (Fig. 3), which represent the majority of the UK's rail bridge population. For these bridges, minor elements of the sub-structure (supports) include wing walls and abutments. Minor elements of the deck comprise the barrel arch, spandrel walls, face rings, and parapets (Network Rail, 2004a).

\subsection{Directed Acyclic Graph}

The minor and major elements can be treated as the nodes (variables) of a BBN. Since a major element's condition is dependent on the condition of its minor elements, the major elements are treated as 'child' variables of its associated minor elements ('parent' variables). Similarly, a bridge condition is a function of the condition of its major elements. Hence, another variable node is introduced in the BBN representing the bridge condition, which is a 'child' of the variables representing the major elements of the bridge. The resulting $\mathrm{BBN}$ model is shown in Fig. 4. For a single span bridge, it consists of 9 variables and 8 links.

The converging connection linking the major and its associated minor elements encodes that minor element conditions are marginally independent, though not if information about the major element condition becomes available. Although the condition of some minor elements can be correlated due to their dependence on some common factors, e.g. surrounding environment 
and live loading, the assumption of marginal conditional independence is not unreasonable as a first approximation on the grounds that:

a) the method of determining the initial distributions for the condition of parent variables through inspection data captures implicitly the dependence on common external factors;

b) there exist micro-climate and spatial effects that can be as important in breaking down the dependence on such common factors;

Each variable of the $\mathrm{BBN}$ is represented through a set of finite condition states. At a single bridge level, such as that proposed by Attoh-Okine and Bowers (2006), a variable can only attain one such state at any given point in time (since the states are mutually exclusive). However, due to the uncertainty in describing the actual condition state, a probability density function can be introduced in a 'degree of belief' approach. At a bridge group/stock level, the probability density function assigned to a variable has a frequency interpretation and can be obtained by collecting inspection results from a sampled group of similar bridges.

\subsection{Condition states for variables of the $B B N$}

In the UK, the condition of bridges owned by Network Rail is represented through a condition marking index, known as Structures Condition Marking Index (SCMI) (Network Rail, 2004a), or more recently termed as 'Bridge Condition Marking Index'. During a detailed inspection of a bridge, typically at 6 year intervals, information on the condition of each minor element is logged by the inspector. An SCMI score, ranging from 0 to 100 , can be derived for the elements using the logged condition (SCMI, 2001). The score of '0' is assigned to an element in extremely poor condition whereas ' 100 ' is assigned to an element in perfect condition. In general, an element having an SCMI score above 80 is considered to be in good condition, with no remedial or repair action required. On the other hand, an element with an SCMI score below 45 is 
considered to be in a poor state, typically in need of some essential maintenance action. Based on this information, each element condition is modelled as a three-state variable, namely: Poor (SCMI range of 0 to 45), Fair (SCMI range of 46 to 80), and Good (SCMI range of 81 to 100).

\subsection{Conditional Probability Tables (CPT) for directed links}

Generally, CPTs for a BBN are derived through the use of expert elicitation, especially where data availability is limited. However, the large number of variables and states in the proposed BBN (Fig. 4) make this task difficult and subjective. For example, 27 conditional probabilities need to be specified for the CPT of the 'support condition' given its parent variables 'abutment condition' and 'wing wall condition', each with three (or more, see later) possible condition states. Altogether, 297 conditional probabilities need to be specified for the three CPTs required. Attempts to reduce these cumbersome requirements have been made, e.g. Celeux et al. (2006) through the adoption of log-linear models and simplifying constraints. An alternative methodology has been devised herein to establish the CPTs for each major element given minor element condition state, based on the knowledge embedded in the creation of a bridge-level SCMI from the scores of minor elements.

Specifically in the SCMI system, each minor element is assigned an element factor in order to reflect its importance in relation to the condition of the linked major element (Network Rail, 2004b). These element factors reflect the background expert knowledge regarding the relative importance of minor elements in defining the condition of a major element and, in turn, a bridge. The element factors currently used for masonry arch bridge elements, and their interpretation as weighting factors, are shown in Table 1.

A conditional probability also captures the strength of the link between a child and its parent variables. Thus, it can be interpreted as a relative weighting factor representing the 
importance of each parent variable condition in establishing the condition of their child variable. For example, in the BBN model of Fig. 4, the support condition depends on the condition of its minor elements, namely abutment and wing walls. Table 1 suggests that the condition of abutment wall matters twice as much as the condition of wing walls in establishing the support condition. Since this is the exhaustive pair of variables affecting support condition, their relative weighting factors will become $2 / 3$ (or 0.67 ) and $1 / 3$ (or 0.33 ) respectively. Hence, in terms of the numerical SCMI scale (0 to 100), the support condition can be expressed as a function of the abutment and wing wall condition as follows

$$
\mathrm{S}_{\mathrm{S}}=0.67 \mathrm{~S}_{\mathrm{A}}+0.33 \mathrm{~S}_{\mathrm{W}}
$$

Eq. 5

where the variables $\mathrm{S}_{\mathrm{S}}, \mathrm{S}_{\mathrm{A}}$, and $\mathrm{S}_{\mathrm{W}}$ represent the SCMI score for the condition of supports, abutments, and wing walls respectively.

Since the parent variables, $\mathrm{S}_{\mathrm{A}}$ and $\mathrm{S}_{\mathrm{W}}$, can attain any $\mathrm{SCMI}$ score from 0 to 100 , it was decided to assign a uniform distribution function ranging from 0 to 100 as a non-informative prior for these variables, and used this to derive the CPT for $\mathrm{S}_{\mathrm{S}}$, i.e.

$$
\mathrm{P}\left(\mathrm{S}_{\mathrm{S}} \mid \mathrm{S}_{\mathrm{A}}, \mathrm{S}_{\mathrm{W}}\right)=\mathrm{P}\left(\mathrm{S}_{\mathrm{S}}, \mathrm{S}_{\mathrm{A}}, \mathrm{S}_{\mathrm{W}}\right) / \mathrm{P}\left(\mathrm{S}_{\mathrm{A}}, \mathrm{S}_{\mathrm{W}}\right)
$$

where the joint probability distribution functions $\mathrm{P}\left(\mathrm{S}_{\mathrm{S}}, \mathrm{S}_{\mathrm{A}}, \mathrm{S}_{\mathrm{W}}\right)$ and $\mathrm{P}\left(\mathrm{S}_{\mathrm{A}}, \mathrm{S}_{\mathrm{W}}\right)$ are estimated through Monte Carlo simulation. The CPT for the support node in the BBN is shown in Table 2 .

Similarly, the conditional probabilities for other variables, i.e. deck and bridge group, have been derived using the relative weighting factors given in Table 1, leading to the relationships shown below

$$
\begin{array}{lr}
\mathrm{S}_{\mathrm{D}}=0.51 \mathrm{~S}_{\mathrm{B}}+0.18 \mathrm{~S}_{\mathrm{F}}+0.13 \mathrm{~S}_{\mathrm{P}}+0.18 \mathrm{~S}_{\mathrm{SW}} & \text { Eq. } 7 \\
\mathrm{~S}_{\mathrm{BG}}=0.5 \mathrm{~S}_{\mathrm{S}}+0.5 \mathrm{~S}_{\mathrm{D}} & \text { Eq. } 8
\end{array}
$$


where, $\mathrm{S}_{\mathrm{D}}=$ Deck condition, $\mathrm{S}_{\mathrm{F}}=$ Face ring condition, $\mathrm{S}_{\mathrm{P}}=$ Parapet condition, $\mathrm{S}_{\mathrm{B}}=$ Barrel arch, $\mathrm{S}_{\mathrm{SW}}=$ Spandrel wall condition, $\mathrm{S}_{\mathrm{S}}=$ Support condition and $\mathrm{S}_{\mathrm{BG}}=$ Bridge condition.

\section{Case Study}

\subsection{Input conditions for masonry arch bridges}

A sample of 50 notionally similar masonry arch bridges was selected to demonstrate the methodology in representing group level condition-based deterioration. The condition state of each element was obtained from the detailed inspection reports of the sampled bridges, yielding a probability distribution for the SCMI score of each minor element in the bridge group. This was used to establish the probabilities associated with each of the three discrete states for the parent variables (minor elements) of the $\mathrm{BBN}$, i.e. $\mathrm{P}($ 'poor') $=\mathrm{P}(0<\mathrm{SCMI} \leq 45), \mathrm{P}$ ('fair') $=$ $\mathrm{P}(46<\mathrm{SCMI} \leq 80)$, and $\mathrm{P}\left({ }^{\prime}\right.$ good' $)=\mathrm{P}(81<\mathrm{SCMI} \leq 100)$ as shown in Table 3.

\subsection{BBN model for masonry arch bridges}

A BBN model representing condition-based deterioration for this group of masonry arch bridges is shown in Fig. 5. The commercially available software 'Hugin Researcher v6.9' is used for the set-up and the ensuing calculations related to this BBN (Jensen and Nielsen, 2007). Since the majority of the minor elements of the chosen sample are in the 'fair' state, the BBN model estimates a higher probability for the major elements (support and deck condition) to be in this state. Similarly, the probability of the bridge group to be in a 'fair' state is significantly higher than the corresponding values for the other two states.

The BBN model was verified through simple checks, using arbitrary extreme input conditions for the minor elements. For example, when all the minor elements are in a single 
state, both the major elements and the bridge group should be expected to be in that same state (Sathananthan, 2010). Moreover, a refined BBN model with five states per element was also constructed by dividing the SCMI 0 to 100 scale into five equal intervals. The results are shown in Fig. 6, and as expected, a more graded condition performance is revealed.

The results of the BBN models were then compared with the computed SCMI distribution for the bridge group using the currently adopted methodology presented in Network Rail (2004a). As shown in Fig. 7, a good overall agreement was observed even with the three-state model, with the small differences (for the lower and upper SCMI ranges) attributed to the assumptions introduced whilst establishing the conditional probability tables between the parent and child variables. It is worth noting that the five-state model requires additional input for the conditional probabilities, which may potentially be difficult to obtain (herein the same methodology was adopted, based on Monte Carlo simulation of uniform non-informative priors). Hence, the number of states in the BBN model should be specified considering the level of precision required, together with any practical constraints in terms of effort and data availability. For illustration purposes, the three-state BBN model is used in the following sections to investigate sensitivities and 'what if' scenarios.

\subsection{Sensitivity Analysis}

A sensitivity analysis was carried out using the BBN model to assess the impact of each minor element on the bridge group condition. The 'what-if' feature described above was used for this purpose. With the BBN model shown in Fig. 5 selected as a reference point, each minor element was assigned, in turn, a probability of ' 1 ' for being in the 'poor' state, i.e. $0<\mathrm{SCMI} \leq 45$, keeping the remaining elements in their existing state and the resulting changes in the bridge group condition were calculated. The sensitivity of bridge group condition on the minor elements 
is computed by normalizing the individually calculated change in the group condition over the total change (i.e. sum of the changes in bridge group conditions from each minor element), and is presented in Fig. 8.

It may be observed that the abutment and barrel arch have the greatest influence in changing the particular bridge group condition corresponding to the input data associated with the BBN in Fig. 6, followed by the wing wall and finally the remaining three minor elements. This ranking is in tune with the prior weightings given to various elements (see Table 1). However, in performing this sensitivity analysis, the initial condition of the minor elements (as recorded on the sample of 50 bridges) also plays a part. It is therefore possible to differentiate between elements that have been assigned nominally equal weightings (e.g. abutment and barrel arch) and thus reveal sensitivities in the light of actual inspection information about a given bridge stock. However, the major limitation of this sensitivity analysis is that the influence of each minor element is investigated separately, which can be misleading in situations where decisions need to be made about the group/stock as a whole. This is demonstrated below.

\subsection{Examples of 'What if' analysis}

A major advantage of the BBN model is that it can be used effectively to analyse 'what-if' scenarios. For example, the expected change in the bridge group condition when new evidence regarding a minor element becomes available, such as new inspection data following a maintenance campaign. The updating methodology described in Sec. 2.1 is used for this purpose. Fig. 9 shows the result from a hypothetical improvement program targeted exclusively on abutments. As can be seen, given the relatively high weighting attributed to this element and its relatively high fraction in 'poor' condition, the effect is to reduce fourfold the fraction of bridges 
falling in 'poor' condition. However, such an exclusive maintenance action is also likely to be unrealistic in actual field situations.

The real power of the BBN as a decision-support tool is in enabling reverse 'what if' analysis to be undertaken, which can lead to the formulation of maintenance plans given desired targets at system (herein group) level. For example, Fig. 10 presents the results for a case where the bridge group condition has been set to a distribution with no 'poor' states and a relatively small percentage of 'good' states. This could be a realistic plan for the infrastructure owner, given the initial condition depicted in Fig. 5. As can be deduced by contrasting the two figures, all minor elements will require improvement, though the relative degree (and hence required effort/resource commitment) is not the same. Wing walls and barrel arches are identified as the two minor elements for which most effort will be required. Comparing this result with what was previously presented in Fig. 8, it is evident that traditional sensitivity analysis can be misleading.

\section{Time dependent condition assessment}

The BBN model discussed in the previous sections serves as a 'snapshot' model to estimate the bridge group condition based on its constituent element conditions at a given point in time. However, deterioration is a time dependent process, hence it is important to be able to model the variation in the bridge condition with time. A useful tool to model such a process is the Dynamic Bayesian Networks (DBN). In a DBN, the basic Bayesian model (termed as 'time slice') is connected to its successive 'time slices' through temporal links to form the time varying model. The relationships between the variables in successive time slices are expressed through conditional probabilities for the temporal links (also known as transition probabilities). The BBN model presented in Sec. 4.2 is transformed schematically into a DBN model as shown in Fig. 11. 
It is worth noting that since only the parent variables are independent variables in a given time slice, only these are linked between successive time slices through the temporal links. The condition for all the child variables, i.e. major elements and the overall bridge condition, are computed using the standard BBN model, developed in Sec. 4, using the condition of parent variables at each time slice.

\subsection{DBN Model for Masonry Arch Bridges}

The input required to define a DBN model, in addition to the information needed to develop a $\mathrm{BBN}$ model, is the transition probabilities related to successive time slices for each minor element. In the absence of any field data, Markov chain principles are introduced to quantify the transitional probabilities. Thus, starting with the BBN model having five discrete states for each variable, a hypothesized scenario is considered that there is a $10 \%$ probability that a variable shifts from its current condition state to the immediately lower condition state at the next time step, e.g. $\mathrm{P}\left(61<\mathrm{Sw}\left(\mathrm{t}_{\mathrm{i}+1}\right) \leq 80 \mid 81<\mathrm{Sw}\left(\mathrm{t}_{\mathrm{i}}\right) \leq 100\right)=0.1$ (This is referred to as $\left.\mathrm{P}_{\mathrm{ii}}\right)$. Hence, according to the Markov chain principle, the probability of wing wall remaining in the same state is 0.9 (i.e. 1 - $\left.\mathrm{P}_{\mathrm{ii}}\right)$. Since, in the absence of interventions, the deterioration process is irreversible, the transition probabilities will, over time, monotonically shift elements from higher to lower states. It is further assumed that when an element reaches the worst possible condition state $\left(0<\operatorname{Sw}\left(\mathrm{t}_{\mathrm{i}}\right) \leq 20\right)$, it can stay in that state indefinitely.

In this process, it is also necessary to specify the transition interval, i.e. the time interval between two successive time steps (i.e. $t_{i+1}-t_{i}$ ). This has been set to six years in order to align with the current practice with respect to the intervals between successive 'principal inspections' followed by many UK bridge authorities but also bearing in mind the variation of SCMI scores with time for the particular type of bridge considered herein. 
The output from the DBN model is the distribution of bridge group condition at various points-in-time. For example, the histograms of $\mathrm{S}_{\mathrm{BG}}$ at time slice 0 (i.e. current condition), and at time slice 1 (i.e. after 6 years), assuming that no maintenance intervention or inspection updating has taken place during this period, are presented in Fig. 12. It clearly illustrates the 'downwards' shift of the distribution of bridge group condition from a mean value of 58.6 (at time slice 0 ) to 50 (at time slice 1), given the deterioration proceeds without any maintenance intervention.

\subsection{Sensitivity analysis}

From the histograms obtained using the DBN model (e.g. Fig. 12), any chosen fractile value for the bridge condition can be used to establish associated condition profiles. As an example, the condition profiles of mean $\mathrm{S}_{\mathrm{BG}}$ are plotted in Fig. 13. Clearly, other fractiles of the overall distribution could also have been selected hence a judicious choice can be made depending on the importance of the particular group on the transport network.

An assumption in the above DBN model is that all minor elements are deteriorating at the same rate, i.e. probabilities for each minor element to maintain their initial state at the next time step, $\mathrm{P}_{\mathrm{ii}}$, is the same. This is not a limitation in the DBN itself but, rather, reflects the lack of available information in this regard. Selective cases have been analysed to demonstrate the DBN's ability to model the scenarios where the deterioration rates are different for various minor elements. These include, e.g. the $\mathrm{P}_{\mathrm{ii}}$ for the abutment as 0.75 whilst maintaining the $\mathrm{P}_{\mathrm{ii}}$ for the rest of minor elements at 0.95 . Similar other cases are analysed for the barrel arch and wing wall and the results are presented in Fig. 13. 


\subsection{Updating Prediction using Inspection Results and Maintenance Effects}

As stated earlier, the BBN model can update the predicted bridge condition when additional information becomes available on the element conditions. The methodology presented in Sec. 2 can be used in the DBN model to identify the expected change in the rate of bridge condition deterioration due to inspection findings and/or any maintenance activities. For example, a scenario may be considered that structural health monitoring or an inspection programme has revealed the abutments to be in 'poor' condition (i.e. $\mathrm{P}\left(0<\mathrm{S}_{\mathrm{A}}<20\right)=1.0$ ) at year 18 (time slice 4). This additional evidence can be formally utilised through Eq. 3 and Eq. 4 to obtain the posterior probability for the bridge condition. Fig. 14a depicts the drop in the mean bridge group condition should the inspection/monitoring outcomes reveal this condition.

Similarly, the effect of a potential maintenance activity can be explored using the DBN model. For example, Fig. 14b indicates the improvement in mean bridge group condition given that the abutments of the group are to be restored to a 'good' condition (i.e. $\mathrm{P}\left(81<\mathrm{S}_{\mathrm{A}} \leq 100\right)=$ 1.0). Other similar scenarios can be readily investigated to aid with management decisions using the modelling techniques presented herein.

\section{Conclusions}

This paper presents the development of a Bayesian Belief Network model aimed at representing bridge condition deterioration. It has been shown that the BBN models are capable of handling complex relationships between bridge elements and the system by means of conditional probabilities specified on a fixed model structure.

The application of the methodology is presented through a case study on the UK's railway masonry arch bridges. The condition of elements obtained from a sample bridge group is used as 
input in the $\mathrm{BBN}$, together with a set of conditional probabilities derived from inspection experience, to yield, in probabilistic terms, the overall group condition. The results from the BBN model are verified using actual data from the sampled bridge group.

A desirable feature of the BBN models is their ability to analyse 'what-if' scenarios. This is particularly helpful for the prioritization of assessment and maintenance activities. This feature has been demonstrated though a sensitivity analysis to obtain the relative importance of each minor element on the overall bridge group condition. This 'what if' capability also makes the BBN approach particularly useful in the context of structural health monitoring, as it would help quantify the value of introducing such tools under different spatial or temporal assumptions.

It is also evident from the results presented that initial element conditions are crucial in determining not only the condition of the particular group of bridges but also in understanding the maintenance/intervention priorities. This, in turn, leads to the question of what inspection/monitoring techniques should be used, how often and with what priority. Once more, the usefulness of the BBN approach lies in its ability to deal with a wide range of scenarios both readily and transparently.

The BBN has been extended to Dynamic Bayesian Network model by introducing the temporal relationship between the parent variables. These are shown to effectively model with time variant deterioration profiles for the group of bridges. It has been demonstrated that the DBN models have the potential to capture the variation in initial conditions of various elements, along with their varying deterioration rates, thus leading to predictions for the deterioration rates for the group of bridges. Their ability to update the deterioration predictions, in the light of data obtainable from inspection, monitoring or maintenance activities, has also been highlighted. 


\section{Acknowledgements}

The authors express their sincere thanks to Network Rail, and in particular Mr Brian Bell, for sponsoring part of the work and for making data available through Mouchel Rail (now SKM). They are also grateful to the partners of the Marie Curie ITN SmartEn for many fruitful discussions and for facilitating a number of interactions that have improved the context and relevance of this work.

\section{References}

Attoh-Okine, N. O. \& Bowers, S. (2006). A Bayesian belief network model for bridge deterioration. Proc. ICE: Bridge Engineering, 159(2), 69-76.

Casciati, F. \& Faravelli, L. (1992). Exploiting expertise in monumental building diagnosis. Paper presented at the meeting of $10^{\text {th }}$ World Conf. Earthquake Eng., Balkema, Rotterdam.

Celeux, G., Corset, F., Lannoy, A., \& Ricard, B. (2006). Designing a Bayesian network for preventative maintenance from expert opinions in a rapid and reliable way. Reliability Engineering and System Safety, 91, 849-856. doi:10.1016/j.ress.2005.08.007

Doguc, O. \& Ramirez-Marquez, J. E. (2009). A generic method for estimating system reliability using Bayesian networks. Reliability Engineering and System Safety, 94 (2), 542-550. doi: 10.1016/j.ress.2008.06.009

Faber, M.H., Kroon, I.B., Kragh, E., Bayly, D., \& Decosemaeker, P. (2002). Risk assessment of decommissioning options using Bayesian networks. Journal of Offshore Mechanics and Arctic Engineering, 124 (4), 231-238. doi: 10.1115/1.1491974 
Friis-Hansen, A. (2000). Bayesian networks as a decision support tool in marine applications. (Unpublished doctoral dissertation), Department of Naval Architecture and Offshore Engineering, Technical University of Denmark, Denmark.

Jensen, F. V. and Nielsen, T. D. (2007). Bayesian networks and decision graphs. Springer.

Khakzad, N., Khan, F., \& Amyotte, P. (2011). Safety analysis in process facilities: Comparison of fault tree and Bayesian network approaches. Reliability Engineering \& System Safety, 96, 925-932. doi: 10.1016/j.ress.2011.03.012

Langseth, H., \& Portinale, L. (2007). Bayesian networks in reliability. Reliability Engineering \& System Safety, 92, 92-108. doi:10.1016/j.ress.2005.11.037

LeBeau, K. \& Wadia-Fascetti, S. (2000). A fault tree model of bridge deterioration. Paper presented at the meeting of $8^{\text {th }}$ ASCE specialty conference on probabilistic mechanics and structural reliability, Indiana, USA.

Marquez, D., Neil, M., \& Fenton, N. (2010). Improved reliability modelling using Bayesian networks and dynamic discretization. Reliability Engineering \& System Safety, 95, 412-425. doi: 10.1016/j.ress.2009.11.012

Murphy, K.P. (2002). Dynamic Bayesian networks: representation, inference and learning. (Unpublished doctoral dissertation), University of California, Berkeley, USA.

Nielsen, J. \& Sørensen, J. D. (2011). Risk-based operation and maintenance of offshore wind turbines using Bayesian networks. Applications of Statistics and Probability in Civil Engineering. Edited by Faber, Kohler, \& Nishijima. 311-317.

Network Rail, (2004a). Structures condition marking index handbook for bridges, NR/GN/CIV/04, Issue 3, Network Rail, UK. 
Network Rail, (2004b). Management of existing bridges and culverts, Ref: NR/SP/CIV/080, Issue 1, Network Rail, UK.

Pearl, J. (1988). Probabilistic reasoning in intelligent systems: Networks of plausible inference. Morgan Kaufmann Publishers.

Rafiq, M.I., Chryssanthopoulos, M.K., \& Onoufriou, T. (2004). Performance updating of concrete bridges using proactive health monitoring systems. Reliability Engineering \& System Safety, 86, 247-256. doi: 10.1016/j.ress.2004.01.012

SCMI (2001). The SCMI algorithm for Railtrack, Taywood Engineering, Unpublished Document no. T207/REP/010.

Sathananthan, S. (2010). Risk-based inspection planning for bridge networks. (Unpublished doctoral dissertation), Faculty of Engineering and Physical Sciences, University of Surrey, UK.

Sathananthan, S., Onoufriou, T., \& Rafiq, M. I. (2008). A risk ranking strategy for network level bridge management. Structure \& Infrastructure Engineering, 6, 767-776. doi: $10.1080 / 15732470802383677$

Sianpur, P. R. M. \& Adams, T. M. (1997). Fault-tree model of bridge element deterioration due to interaction. Journal of Infrastructure Systems, 3, 103-110. doi: 10.1061/(ASCE)10760342(1997)3:3(103)

Straub, D. (2009). Stochastic Modelling of deterioration processes through Dynamic Bayesian Networks. Journal of Engineering Mechanics, 1089-1099. doi:10.1061/(ASCE)EM.19437889.0000024 
Weber, P. \& Jouffe, L., (2003). Reliability modelling with dynamic Bayesian networks, presented at the 5th IFAC Symposium on Fault Detection, Supervision and Safety of Technical Processes, Washington D.C. 


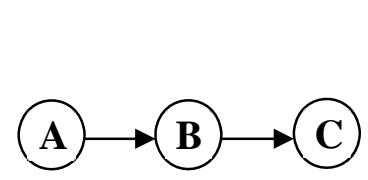

(a). Serial

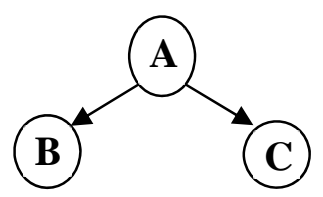

(b). Diverging

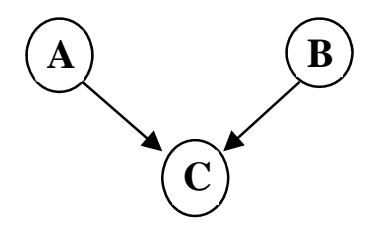

(c). Converging

Fig. 1: Connection types in a BBN

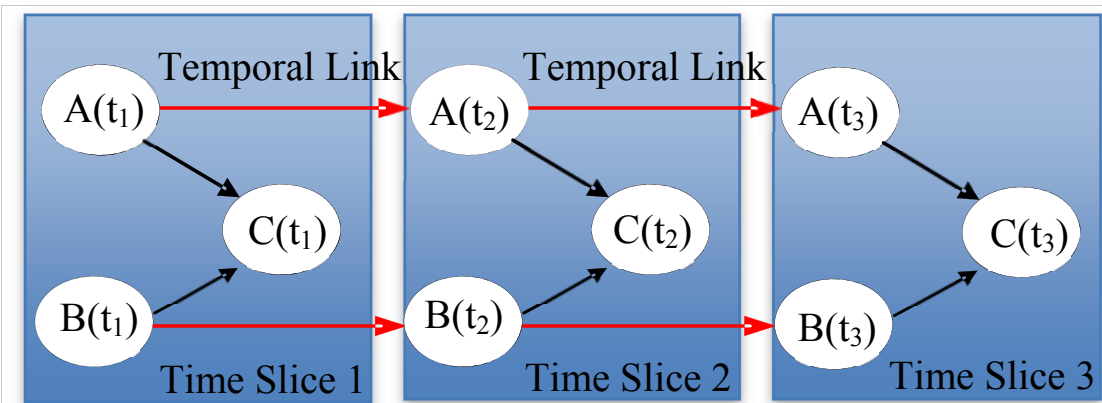

Fig. 2: A DBN example

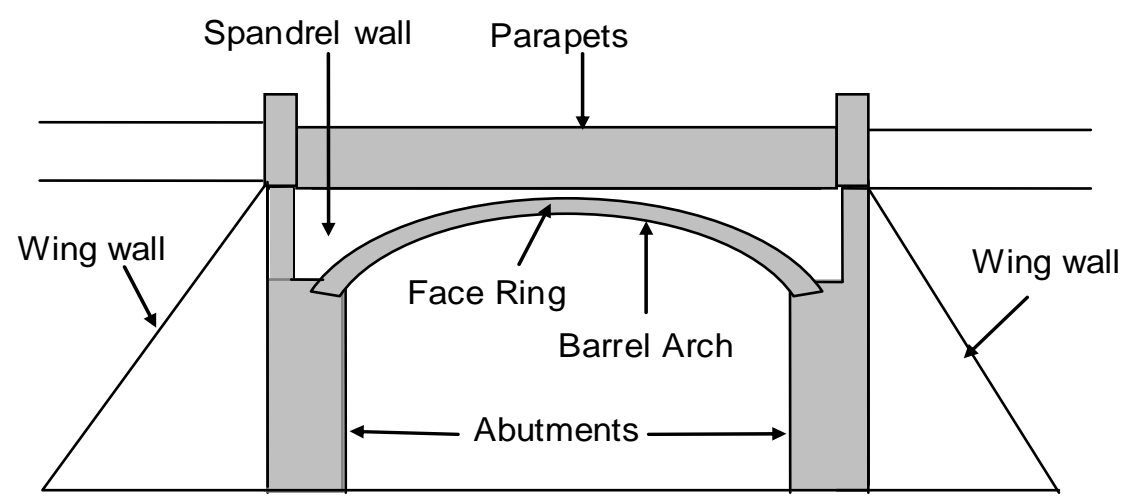

Fig. 3: Typical minor elements of a masonry arch bridge 


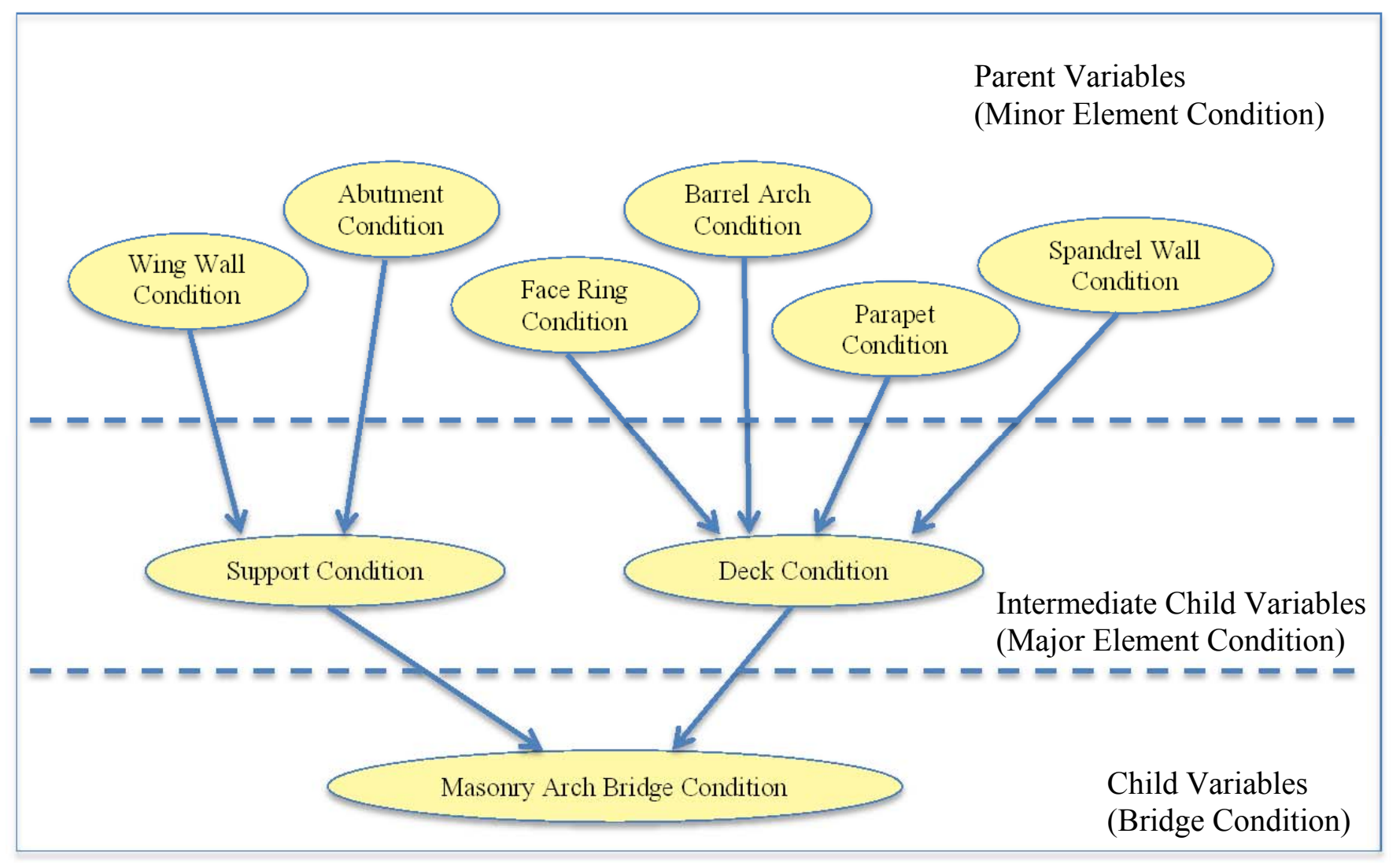

Fig. 4: A BBN for masonry arch bridges

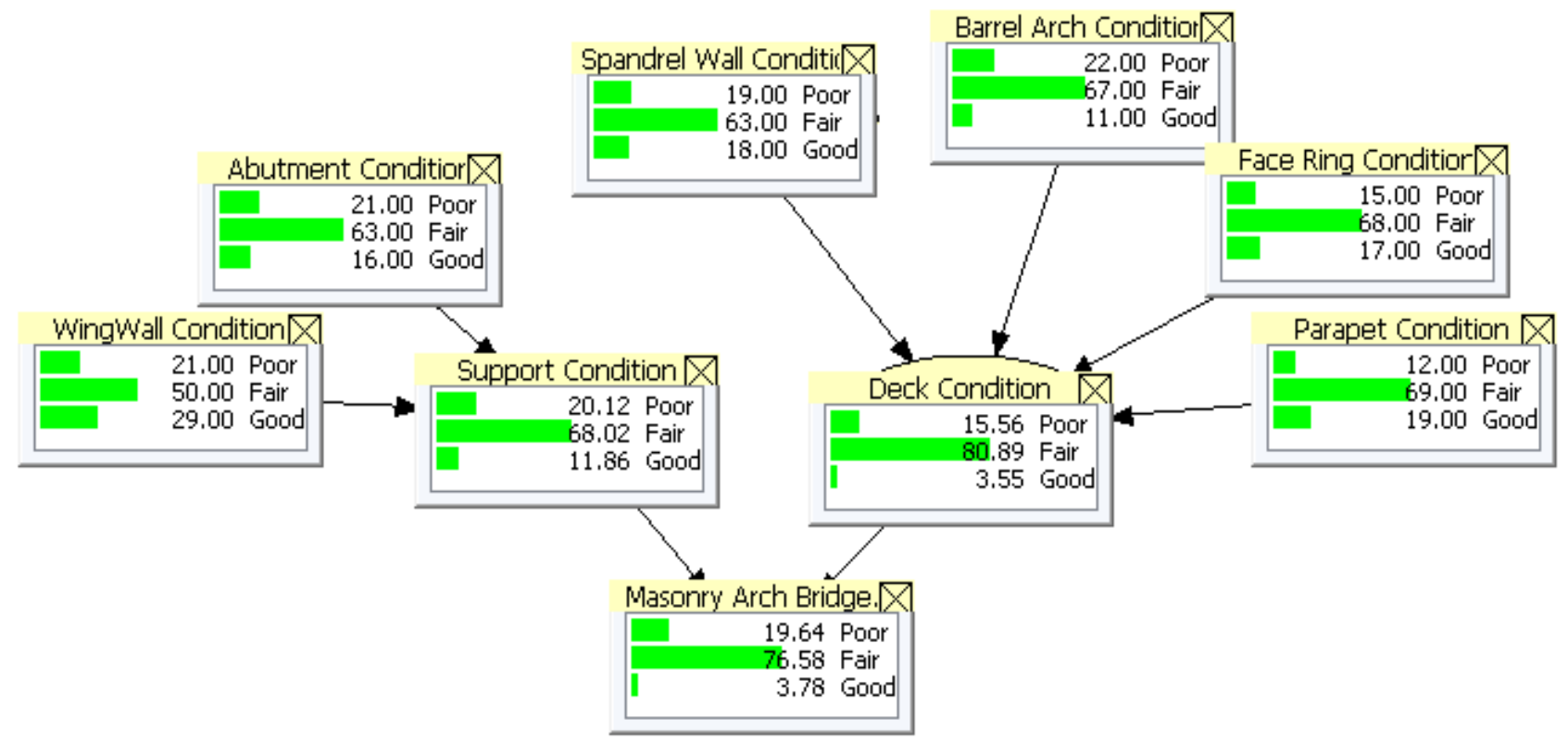

Fig. 5: Three-state BBN model for deterioration of masonry arch bridge group. 


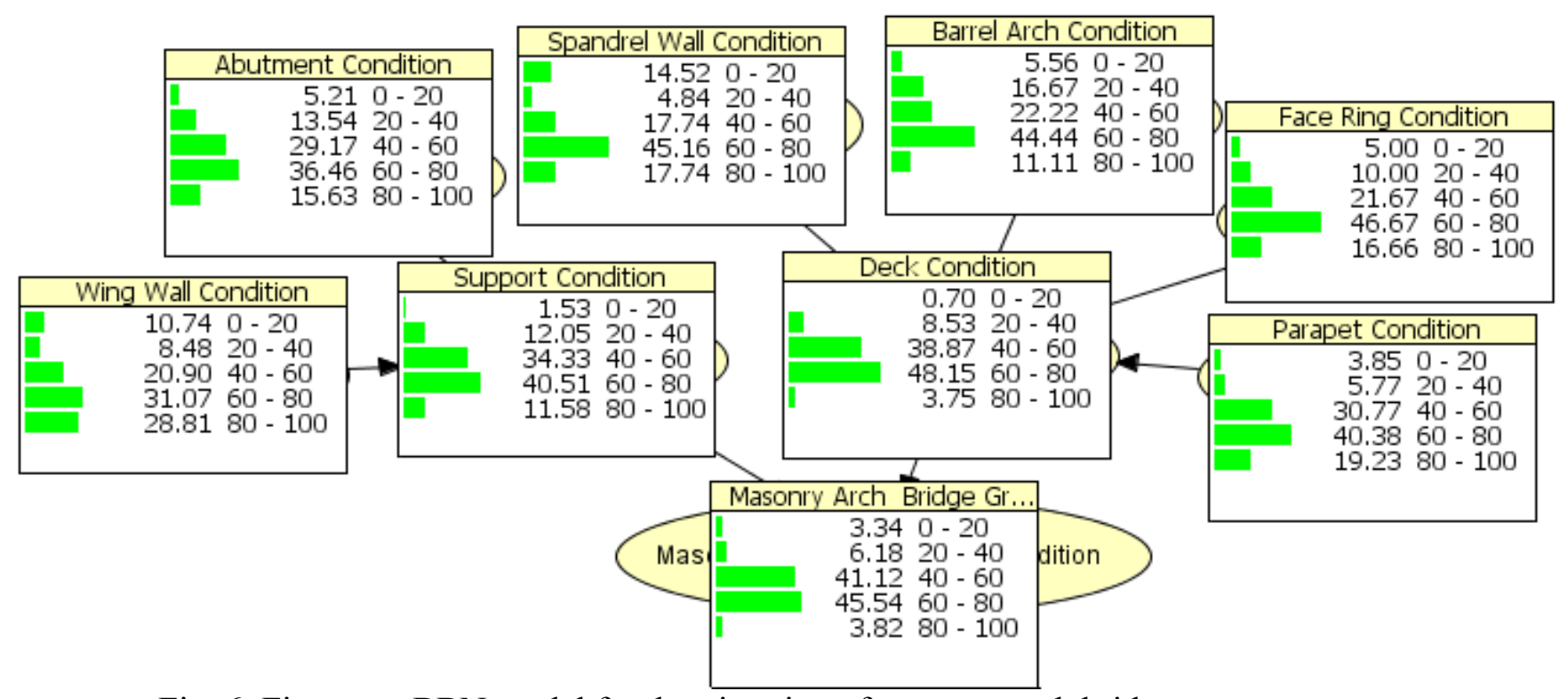

Fig. 6: Five-state BBN model for deterioration of masonry arch bridge group.

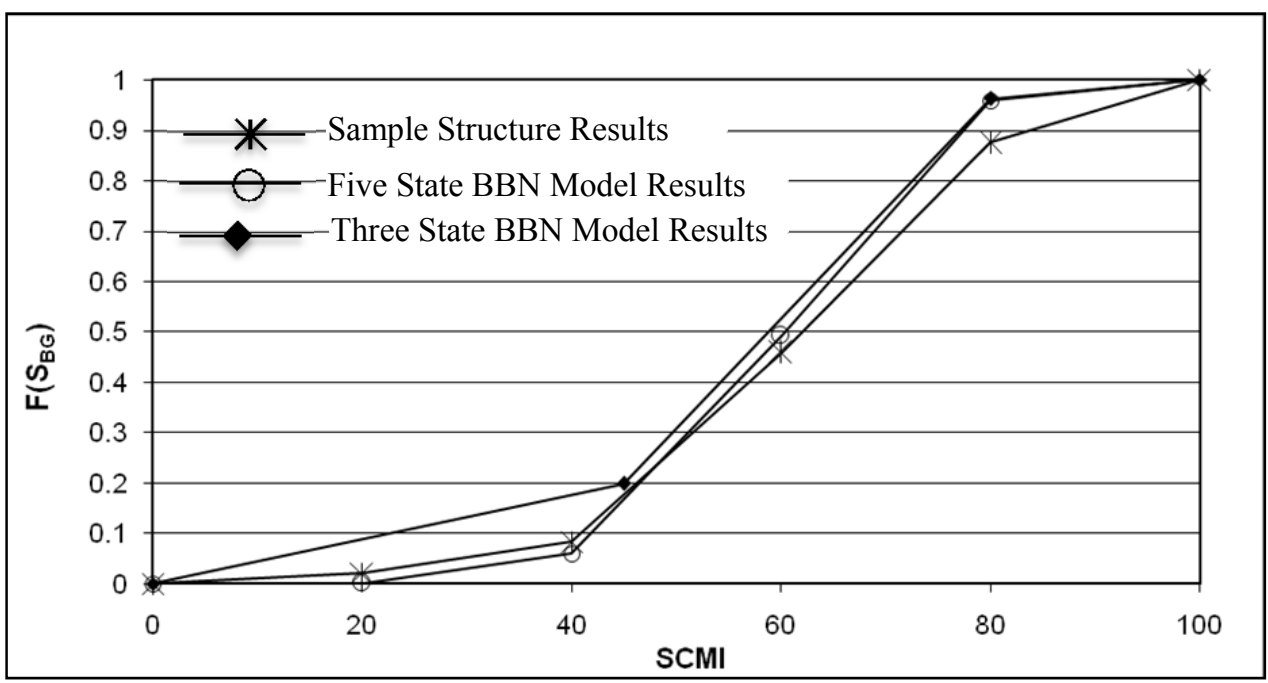

Fig. 7: Comparison of three- and five-state models with SCMI distribution 


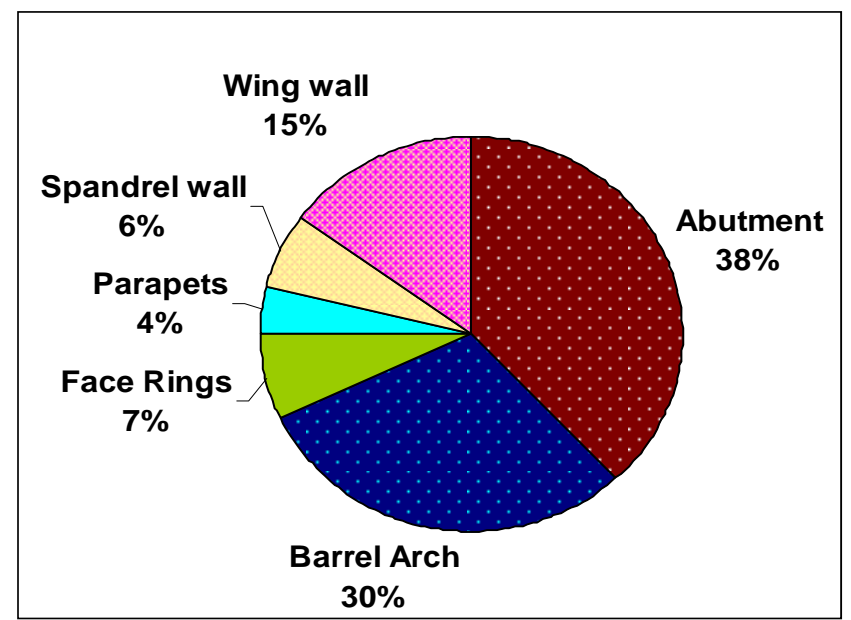

Fig. 8: Sensitivity of a given bridge group condition on minor elements.

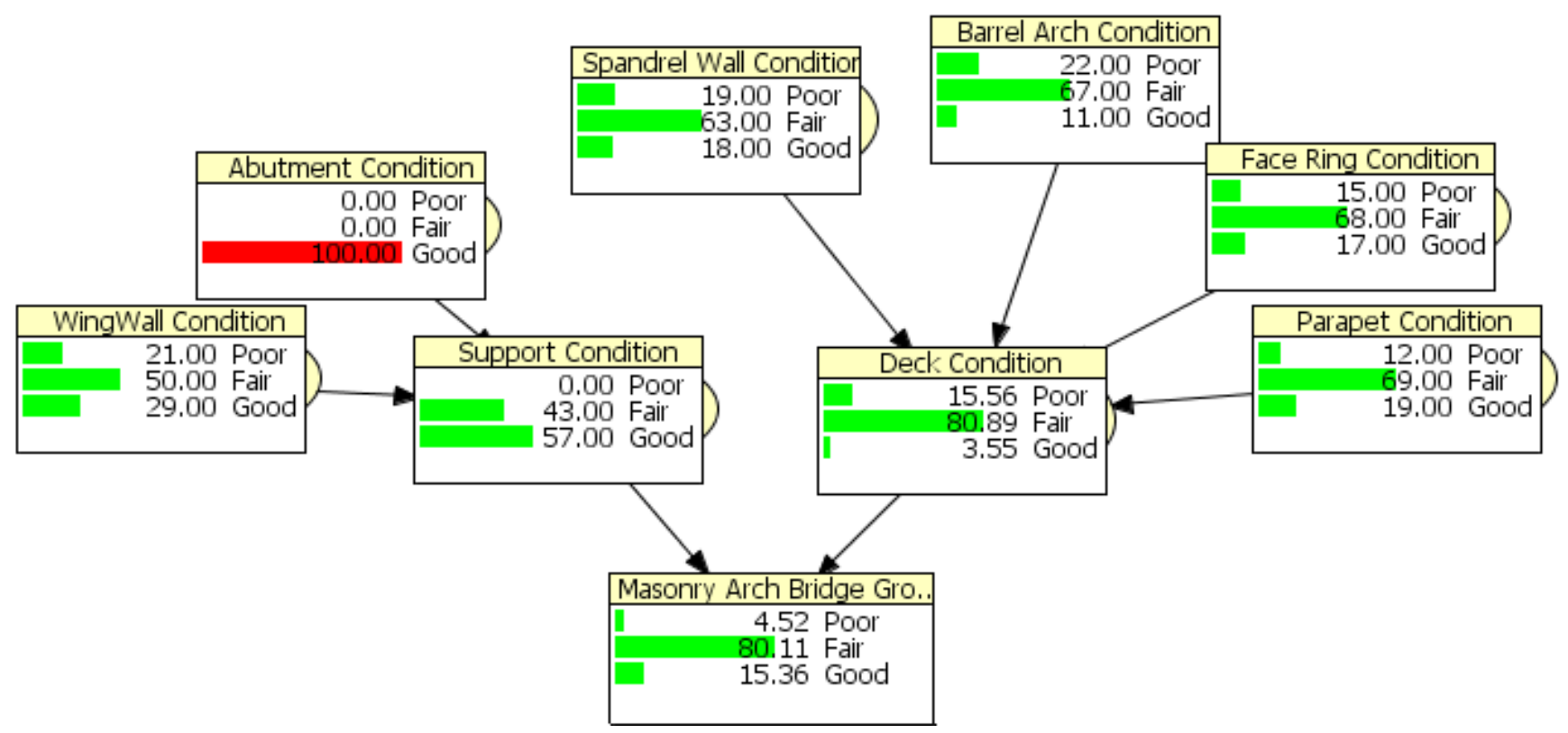

Fig. 9: An example of forward 'what-if' scenario 


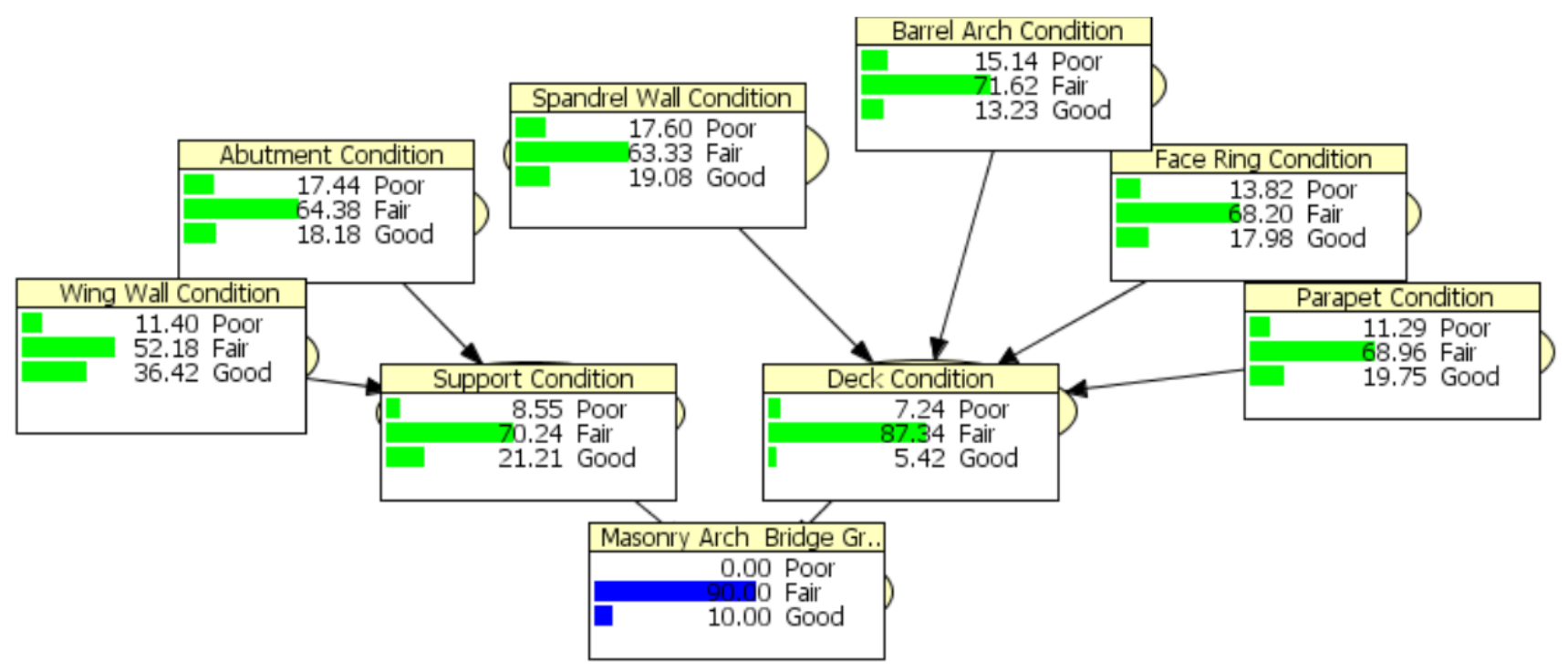

Fig. 10: An example of reverse 'what-if' scenario

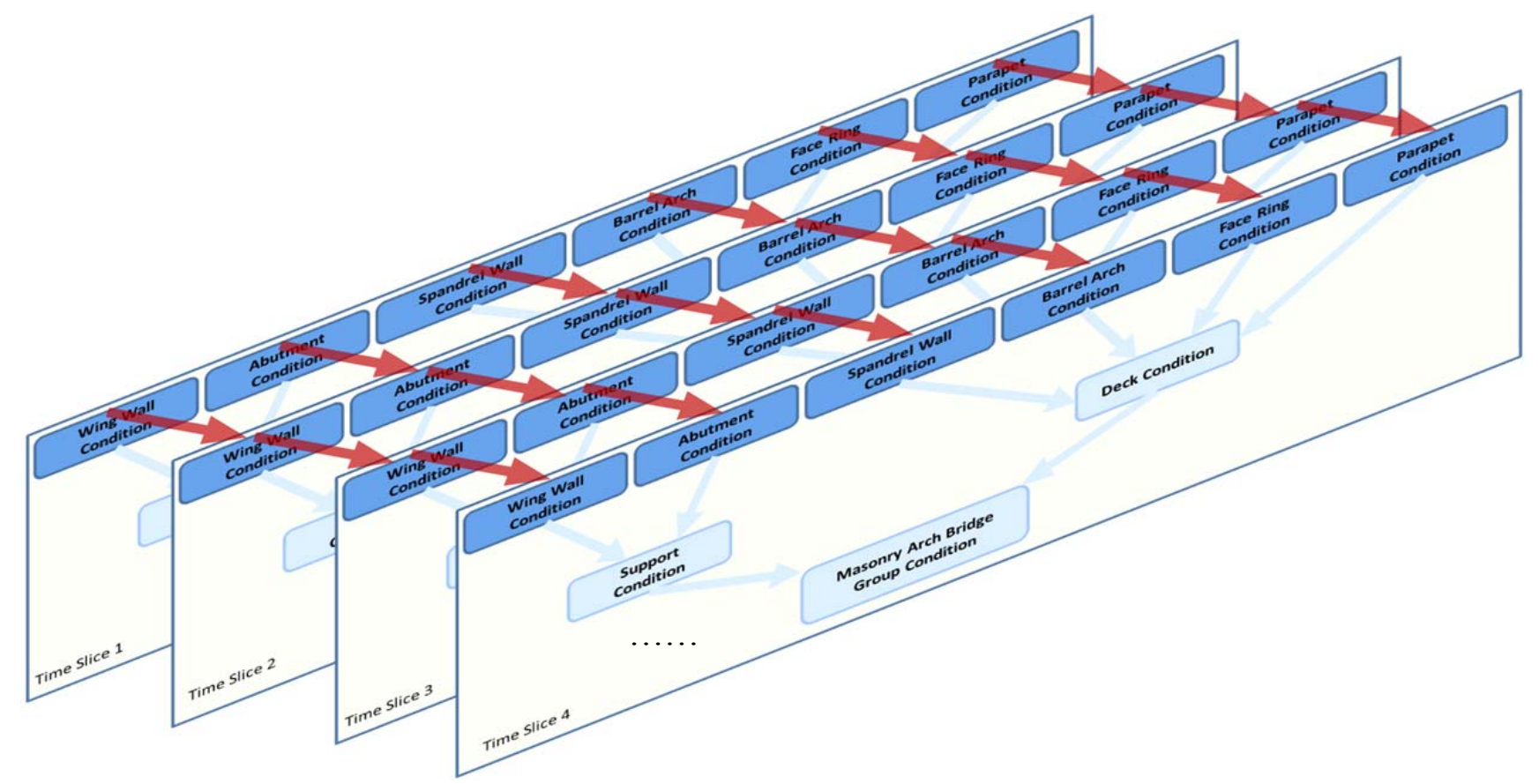

Fig. 11: A DBN model for masonry arch bridge condition 


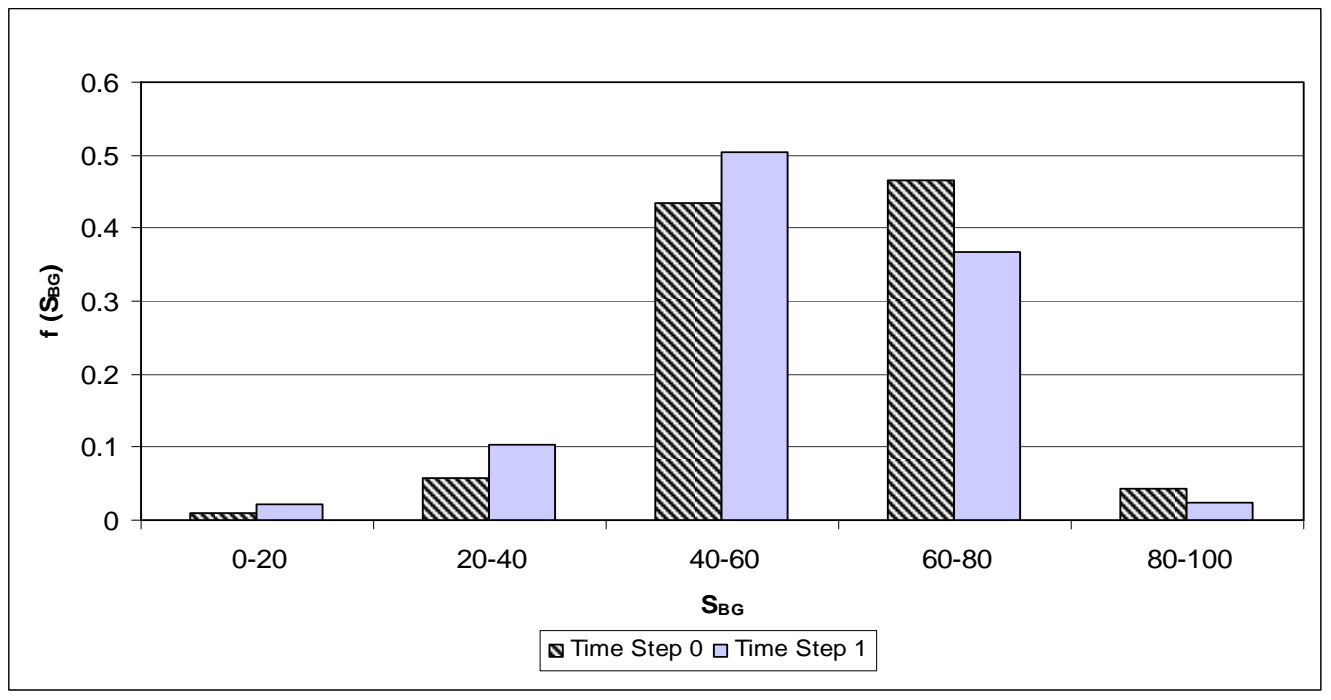

Fig. 12: Change in $\mathrm{S}_{\mathrm{BG}}$ from 'time step 0' to 'time step 1'

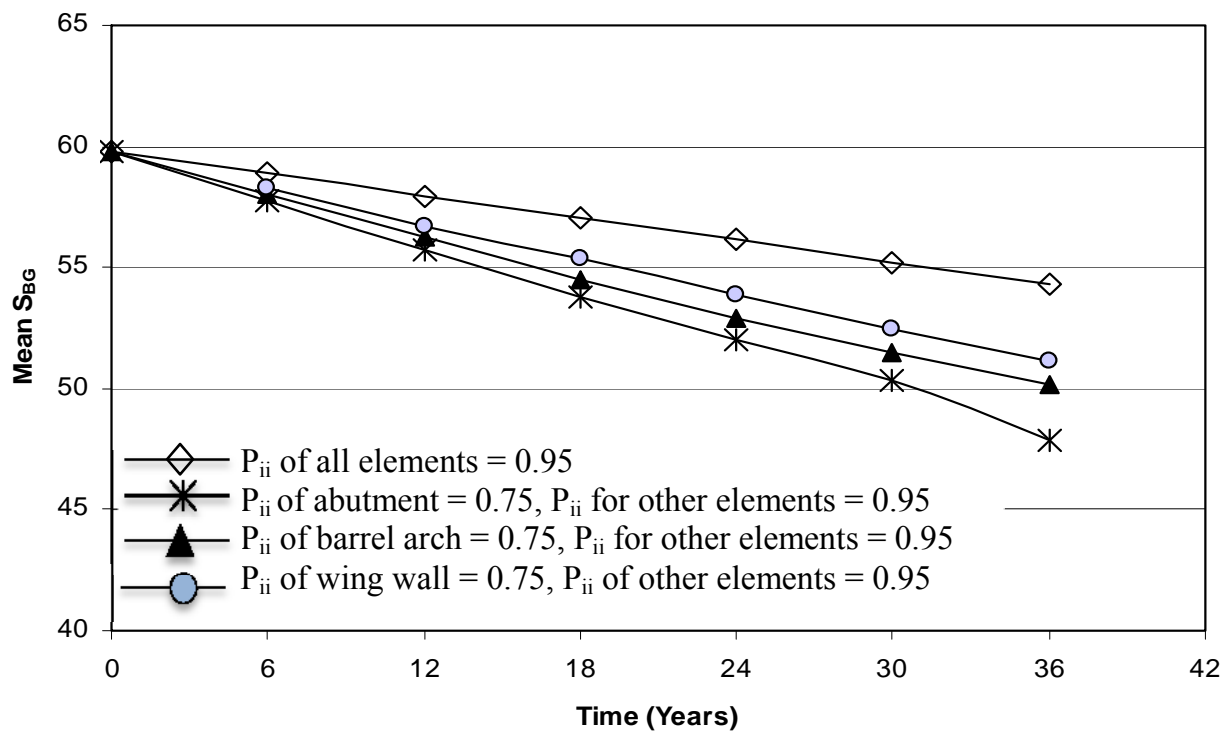

Fig. 13: Change in $\mathrm{S}_{\mathrm{BG}}$ with different element deterioration rates 

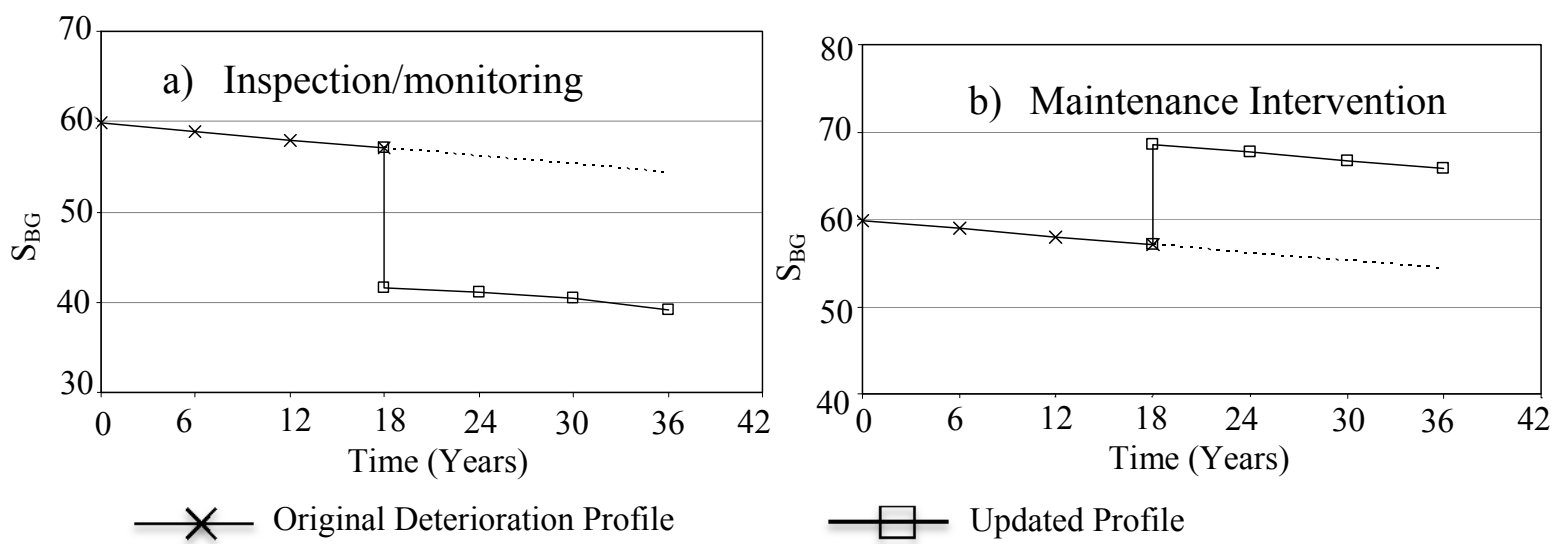

Fig. 14: Updated deterioration profiles

Table 1: Element factors for minor elements (SCMI, 2001)

\begin{tabular}{|c|l|c|l|}
\hline Major Element & Minor Element & $\begin{array}{l}\text { Element } \\
\text { Factor }\end{array}$ & $\begin{array}{l}\text { Relative } \\
\text { Weighting }\end{array}$ \\
\hline \multirow{2}{*}{ Support } & Abutment & 10 & $0.67(=10 / 15)$ \\
\cline { 2 - 4 } & Wing wall & 5 & $0.33(=5 / 15)$ \\
\hline \multirow{5}{*}{ Deck } & Barrel Arch & 10 & $0.51(=10 / 19.5)$ \\
\cline { 2 - 4 } & Face Rings & 3.5 & $0.18(=3.5 / 19.5)$ \\
\cline { 2 - 4 } & Parapets & 2.5 & $0.13(=2.5 / 19.5)$ \\
\cline { 2 - 4 } & Spandrel wall & 3.5 & $0.18(=3.5 / 19.5)$ \\
\hline
\end{tabular}


Table 2: CPT for the Support node

\begin{tabular}{|c|c|c|c|c|}
\hline \multirow{2}{*}{$\begin{array}{c}\text { Wing wall } \\
\text { Condition }\end{array}$} & \multirow{2}{*}{$\begin{array}{c}\text { Abutment } \\
\text { Condition }\end{array}$} & \multicolumn{3}{|c|}{ Support Condition } \\
\cline { 3 - 5 } & Poor & 1 & \multicolumn{1}{c|}{ Fair } & Good \\
\hline \multirow{3}{*}{ Poor } & Fair & 0.33 & 0.67 & 0 \\
\cline { 2 - 5 } & Good & 0 & 1 & 0 \\
\cline { 2 - 5 } & Poor & 0.79 & 0.21 & 0 \\
\hline \multirow{3}{*}{ Fair } & Fair & 0 & 1 & 0 \\
\cline { 2 - 5 } & Good & 0 & 0.44 & 0.56 \\
\cline { 2 - 5 } & Poor & 0.50 & 0.50 & 0 \\
\hline \multirow{3}{*}{ Good } & Fair & 0 & 0.85 & 0.15 \\
\cline { 2 - 5 } & Good & 0 & 0 & 1 \\
\cline { 2 - 5 } & & & & 0.8 \\
\hline
\end{tabular}

Table 3: Probabilities for minor elements being in any given condition state.

\begin{tabular}{|l|c|c|c|}
\hline Element type & Poor & Fair & Good \\
\hline Wing wall & 0.21 & 0.50 & 0.29 \\
\hline Abutment & 0.21 & 0.63 & 0.16 \\
\hline Barrel Arch & 0.22 & 0.67 & 0.11 \\
\hline Face Ring & 0.15 & 0.68 & 0.17 \\
\hline Parapets & 0.12 & 0.69 & 0.19 \\
\hline Spandrel wall & 0.19 & 0.63 & 0.18 \\
\hline
\end{tabular}

